\title{
SECOND-ORDER EHRENFEUCHT GAMES AND THE DECIDABILITY OF THE SECOND-ORDER THEORY OF AN EQUIVALENCE RELATION
}

\author{
RICHARD L. TENNEY
}

(Received 19 November 1973; revised 19 May 1974)

Communicated by J. N. Crossley

\begin{abstract}
In this paper we extend a method of Ehrenfeucht and Fraissé to second-order theories and use this extension to prove the decidability of the second-order theory of an equivalence relation.
\end{abstract}

\section{Introduction}

Janiczak (1953) shows that the first-order theory of a single equivalence relation is decidable but the first-order theory of two equivalence relations is not. In this paper, we show that the monadic second-order theory of a single equivalence relation is decidable. We do this using an extension of a technique of Ehrenfeucht and Fraissé. It should be noted that it is possible to encode this theory in the monadic second order theory of two successors for which Rabin (1969) provides a decision procedure. Our techniques have the advantages of being both direct and perspicuous: we show that to decide a sentence in the theory, one must investigate its validity in only a finite number of finite models, and we give primitive recursive bounds on both these numbers.

This paper comprises two parts: an exposition of the extension of first-order Ehrenfeucht games to second-order, a subject of interest in its own right, followed by an application of this technique to the second-order theory of an equivalence relation.

\section{Second-Order Ehrenfeucht games}

We extend the technique of Ehrenfeucht and Fraissé described in Ehrenfeucht (1961), a technique referred to as Ehrenfeucht games. A relational system, $\mathfrak{M}=\left\langle\Omega, R_{i}\right\rangle_{i \in I}$ (with subscripts, as appropriate), of type $v$ is a non-empty set

This paper is based on part of the author's Ph.D. thesis, completed at Cornell University under the direction of Anil Nerode. 
$\Omega$ together with relations indexed by a set $I$, in which $R_{i} \subseteq \Omega^{v(i)}$, where $v: I \rightarrow \omega$ and $\omega=\{0,1,2, \cdots\}$. A relational system of type $\nu$ is finitary if the domain of $v$ is finite (i.e., if there are only a finite number of relations). Two relational systems are similar if they are of the same type. Let $\mathfrak{M}=\left\langle\Omega, R_{i}\right\rangle_{i \in I}$. If $\eta_{j} \subseteq \Omega$ for each $j$ in some set $J$, then the relational system $\left\langle\mathfrak{M}, \eta_{j}\right\rangle_{j \in J}=$ $\left\langle\Omega, R_{i}, \eta_{j}\right\rangle_{i \in I, j \in J}$, which is the relational system obtained frcm $\mathfrak{M}$ by adjoining the unary relations $\eta_{j}(j \in J)$. Similarly, if $y_{k} \in \Omega$ for each $k$ in some set $K$, then $\left\langle\mathfrak{M}, y_{k}\right\rangle_{k \in K}=\left\langle\Omega, R_{i}, y_{k}\right\rangle_{i \in I, k \in K}$, which is the relational system with certain elements distinguished. We will abuse notation and write $\eta \subseteq \mathfrak{M}$ for $\eta \subseteq \Omega$ and $y \in \mathfrak{M}$ for $y \in \Omega$. By "integer" we always mean "non-negative integer" where $0=\varnothing$ and $n+1=\{n\} \bigcup n$.

Assume $\mathfrak{M}_{0}$ and $\mathfrak{M}_{1}$ are similar relational systems and $m, n \in \omega$. The secondorder Ehrenfeucht game $\mathfrak{G}_{m, n}\left(\mathfrak{M}_{0}, \mathfrak{M}_{1}\right)$ is played as follows. There are two players, player I and player II, and each player makes $m+n$ moves. For his first $m$ moves, each player selects subsets of $\mathfrak{M}_{0}$ and $\mathfrak{M}_{1}$; for the remaining $n$ moves, he selects elements of $\mathfrak{M}_{0}$ and $\mathfrak{M}_{1}$. Specifically, at move $j$, for $j<m$, player I selects one of the two relational systems $\left(\mathfrak{M}_{0}\right.$ or $\left.\mathfrak{M}_{1}\right)$ and a subset of that system. Then player II selects a subset of the other relational system. Denote by $\eta_{j}$ the subset of $\mathfrak{M}_{0}$ chosen at move $j$, regardless of which player chose it; similarly, $\theta_{j} \subseteq \mathfrak{M}_{1}$. At move $j$, for $m \leqq j<m+n$, player I selects one of the two relational systems and an element from that system. Then player II selects an element from the other relational system. Denote by $x_{j-m}$ the element of $\mathfrak{M}_{0}$ chosen at move $j$; similarly, $y_{j-m} \in \mathfrak{M}_{1}$.

Player II wins the game $\mathfrak{G}_{m, n}\left(\mathfrak{M}_{0}, \mathfrak{M}_{1}\right)$ if and only if at the end of the game, the map $\mu: X=\left\{x_{k} \mid k<n\right\} \rightarrow Y=\left\{y_{k} \mid k<n\right\}$ defined by $\mu\left(x_{k}\right)=y_{k}$ for $k<n$ is an isomorphism with respect both to the relations of $\mathfrak{M}_{0}$ and $\mathfrak{M}_{1}$ (restricted to $X$ and $Y$ respectively) and to the chosen subsets (also restricted to $X$ and $Y$ ). Of particular interest are those pairs of relational systems $\mathfrak{M}_{0}$ and $\mathfrak{M}_{1}$ such that player II can always win the game $\mathfrak{G}_{m, n}\left(\mathfrak{M}_{0}, \mathfrak{M}_{1}\right)$. In order to capture this notion, we inductively define two sequences of equivalence relations, $\equiv_{n}$ and $\equiv_{m, n}$, between similar relational systems with designated elements. The first is that of Ehrenfeucht (1961).

DefinItion 1. $\left\langle\mathfrak{M}_{0}, x_{i}\right\rangle_{i<k} \equiv_{0}\left\langle\mathfrak{M}_{1}, y_{i}\right\rangle_{i<k}$ if and only if the map $\mu: X=$ $\left\{x_{i} \mid i<k\right\} \rightarrow Y=\left\{y_{i} \mid i<k\right\}$ defined by $\mu\left(x_{i}\right)=y_{i}$, for $i<k$, is an isomorphism with respect to the relations of $\mathfrak{M}_{0}$ and $\mathfrak{M}_{1}$ restricted to $X$ and $Y$ respectively. $\left\langle\mathfrak{M}_{0}, x_{i}\right\rangle_{i<k} \equiv_{n+1}\left\langle\mathfrak{M}_{1}, y_{i}\right\rangle_{i<k}$ if and only if for each $x_{k} \in \mathfrak{M}_{0}$ there is a $y_{k} \in \mathfrak{M}_{1}$, and for each $y_{k} \in \mathfrak{M}_{1}$ there is an $x_{k} \in \mathfrak{M}_{0}$ such that $\left\langle\mathfrak{M}_{0}, x_{i}\right\rangle_{l<k+1} \equiv_{n}\left\langle\mathfrak{M}_{1}, y_{i}\right\rangle_{i<k+1}$. For $<\mathfrak{M}_{0}, x_{i}>_{i<0} \equiv_{n}<\mathfrak{M}_{1}, y_{i}>_{i<0}$ write $\mathfrak{M}_{0} \equiv{ }_{n} \mathfrak{M}_{1}$.

Definition 2. $\left\langle\mathfrak{M}_{0}, \eta_{i}\right\rangle_{i<k} \equiv_{0, n}\left\langle\mathfrak{M}_{1}, \theta_{i}\right\rangle_{i<k}$ if and only if $\left\langle\mathfrak{M}_{0}, \eta_{i}\right\rangle_{i<k} \equiv_{n}$ $\left\langle\mathfrak{M}_{1}, \theta_{i}\right\rangle_{i<k} ;$ and $\left\langle\mathfrak{M}_{0}, \eta_{i}\right\rangle_{i<k} \equiv_{m+1, n}\left\langle\mathfrak{M}_{1}, \theta_{i}\right\rangle_{i<k}$ if and only if for each $\eta_{k} \subseteq \mathfrak{M}_{0}$
there is a $\theta_{k} \subseteq \mathfrak{M}_{1}$, and for each $\theta_{k} \subseteq \mathfrak{M}_{1}$ there is an $\eta_{k} \subseteq \mathfrak{M}_{0}$ such that 
$\left\langle\mathfrak{M}_{0}, \eta_{i}\right\rangle_{i<k+1} \equiv_{m, n}\left\langle\mathfrak{M}_{1}, \theta_{i}\right\rangle_{i<k+1}$. As above, write $\mathfrak{M}_{0} \equiv_{m, n} \mathfrak{M}_{1}$ for the case $k=0$.

It is easily verified that these are indeed equivalence relations. Furthermore, it is readily apparent that player II can always win the game $\mathfrak{G}_{m}\left(\mathfrak{M}_{0}, \mathfrak{M}_{1}\right)$ after the $k$ moves $\eta_{0} \leftrightarrow \theta_{0}, \cdots, \eta_{k-1} \leftrightarrow \theta_{k-1}$ if and only if $\left\langle\mathfrak{M}_{0}, \eta_{j}\right\rangle_{j<k} \equiv_{m-k, n}\left\langle\mathfrak{M}_{1}, \theta_{j}\right\rangle_{j<k}$

The importance of this notion derives from the following two theorems. We omit the proofs of these theorems as they are virtually identical with the proofs of the corresponding theorems given in Ehrenfeucht (1961). The reader wanting more details is referred to LeTourneau (1968). The development of second-order Ehrenfeucht games in LeTourneau's unpublished thesis (1968) was prior to (but independent of) ours which was begun along lines suggested by Anil Nerode. We were informed of LeTourneau's work before we had gone much beyond the basic concepts and theorems and a few simple examples. Unlike him, we did not demand that the moves in which sets are chosen precede the moves in which elements are chosen, but we adopt his convention here as it yields slightly simpler definitions and proofs.

We use the usual convention that all the free variables of the formula $\phi\left(\xi_{0}, \cdots, \xi_{m-1}, v_{0}, \cdots, v_{n-1}\right)$ are found among $\xi_{0}, \cdots, \xi_{m-1}, v_{0}, \cdots, v_{n-1}$. In addition, $\xi_{j}$ is understood to be a monadic second-order variable, while $v_{j}$ is a first-order variable; furthermore, $Q_{j}$ represents a monadic second-order quantifier, and $Q_{j}$ represents a first-order quantifier. The notation $\mathfrak{M} \vDash \phi\left[\eta_{0}, \cdots, \eta_{m-1}, x_{0}, \cdots, x_{n-1}\right]$ means that $\phi$ holds in the relational system $\mathfrak{M}$ (equivalently, that $\mathfrak{M}$ is a model for $\phi$ ) when $\xi_{i}$ is interpreted as the subset $\eta_{i} \subseteq \mathfrak{M}$, for $i<m$, and $v_{j}$ is interpreted as the element $x_{j} \in \mathfrak{M}$, for $j<n$.

THEOREM 1. Let $\psi\left(\xi_{0}, \cdots, \xi_{k-1}\right)=Q_{k} \xi_{k} \cdots Q_{m-1} \xi_{m-1} Q_{0} v_{0} \cdots Q_{n-1} v_{n-1} \phi\left(\xi_{0}, \cdots\right.$, $\left.\xi_{m-1}, v_{0}, \cdots, v_{n-1}\right)$, where $\phi$ is quantifier free. If $\left\langle\mathfrak{M}_{0}, \eta_{j}\right\rangle_{j<k} \equiv_{m-k . n}\left\langle\mathfrak{M}_{1}, \theta_{j}\right\rangle_{j<k}$, then $\mathfrak{M}_{0} \vDash \psi\left[\eta_{0}, \cdots, \eta_{k-1}\right]$ if and only if $\mathfrak{M}_{1} \vDash \psi\left[\theta_{0}, \cdots, \theta_{k-1}\right]$.

THEOREM 2. For every finitary relational system $\mathfrak{M}_{0}$ and every set of subsets $\left\{\eta_{j} \subseteq \mathfrak{M}_{0} \mid j<k\right\}$, there is a formula $\chi\left(\xi_{0}, \cdots, \xi_{k-1}\right)$ such that for any finitary relational system $\mathfrak{M}_{1}$ and any set of subsets $\left\{\theta_{j} \subseteq \mathfrak{M}_{1} \mid j<k\right\}$, $\left\langle\mathfrak{M}_{0}, \eta_{j}\right\rangle_{j<k} \equiv_{m-k, n}\left\langle\mathfrak{M}_{1}, \theta_{j}\right\rangle_{j<k}$ if and only if $\mathfrak{M}_{1} \vDash \chi\left[\theta_{0}, \cdots, \theta_{k-1}\right]$.

\section{Decidability of an equivalence relation}

Assume that all relational systems mentioned below are models for the theory of an equivalence relation $\sim$ in which the axioms state that $\sim$ is reflexive, symmetric, and transitive. Define $[z]=\{y \mid y \sim z\}$. The cardinality of a set $\rho$ will be denoted $\#(\rho)$ or simply $\# \rho$.

Assume $m$ and $n$ are fixed. The essence of the proof is a combinatorial analysis of what information about the cardinalities of equivalence classes of a model 
$\mathfrak{M}_{0}$ is necessary to find a finite model $\mathfrak{M}_{1}$ such that player II may always win the second-order Ehrenfeucht game $\mathfrak{G}_{m, n}\left(\mathfrak{M}_{0}, \mathfrak{M}_{1}\right)$. To perform this analysis we introduce several equivalence relations which, of course, should not be confused with the equivalence relation $\sim$.

DEFINITION 3. For each integer $n \in \omega$, define an equivalence relation $E_{n}$ on $\omega+1$ as follows: $k_{0} E_{n} k_{1}$ if and only if $k_{0}=k_{1}$ or both $k_{0} \geqq n$ and $k_{1} \geqq n$.

DEFINITION 4. For each integer $n \in \omega$ define an equivalence relation $\mathscr{E}_{n}$ on models as follows: $\mathfrak{M}_{0} \mathscr{E}_{n} \mathfrak{M}_{1}$ if and only if for each $k<n, C_{0}(k) E_{n} C_{1}(k)$ and $\left[\Sigma_{k \geqq n} C_{0}(k)\right] E_{n}\left[\Sigma_{k \geqq n} C_{1}(k)\right]$, where $C_{i}(k)$ is the number of equivalence classes of cardinality $k$ in $\mathfrak{M}_{i}(i=0,1)$, and the sums are defined to be $\omega$ if they are not finite.

We wish to examine in some detail the cardinality of the intersection of an equivalence class with each of the $2^{m}$ Boolean combinations of $m$ sets. The amount of detail is characterized by the equivalence relation $E_{n}$. To do this we introduce some notation. For a set $\rho, \rho^{0}=\rho$ and $\rho^{1}=\bar{\rho}=$ complement of $\rho$. Enumerate functions from an integer $k$ to $\{0,1\}$ in such a way that $f_{i}^{(k)}(n)$ is the digit in position $n$ (numbering from left to right) in the $k$ digit binary representation of $i$. Write $f_{i}$ for $f_{i}^{(k)}$ when $k$ is clear from context. Note that $f_{2 i}^{(k+1)}$ and $f_{2 i+1}^{(k+1)}$ both extend $f_{i}^{(k)}$. Assume that for $j<m, \eta_{j} \subseteq \mathfrak{M}_{0}$ and $\theta_{j} \subseteq \mathfrak{M}_{1}$. Use the functions $f_{l}^{(m)}$, for $l<2^{m}$, to define the partition of cardinality $2^{m}$ determined by each of these systems of sets. Thus for $l<2^{m}$, set $\Pi_{0}(l)=\bigcap_{j<m} \eta_{j}^{f_{1}^{\prime}(j)}$ and $\Pi_{1}(l)=$ $\bigcap_{1<m} \theta_{j}^{f^{\prime}(j)}$. Let Seq $(k, n)$ represent the set of sequences of length $k$ with elements from 0 to $n$. define

Definition 5. Given $\eta_{j} \subseteq \mathfrak{M}_{0}$ and $\theta_{j} \subseteq \mathfrak{M}_{1}$, for $j<m$, for each $\boldsymbol{i} \in \operatorname{Seq}\left(2^{m}, n\right)$

and

$$
\begin{aligned}
& \gamma_{0}(i)=\left\{[x] \subseteq \mathfrak{M}_{0} \mid \forall l<2^{m}\left[\#\left([x] \cap \Pi_{0}(l)\right) E_{n} i(l)\right]\right\}, \\
& \gamma_{1}(i)=\left\{[y] \subseteq \mathfrak{M}_{1} \mid \forall l<2^{m}\left[\#\left([y] \cap \Pi_{1}(l)\right) E_{n} i(l)\right]\right\} .
\end{aligned}
$$

Note that for $\boldsymbol{i}, \boldsymbol{j} \in \operatorname{Seq}\left(2^{m}, n\right)$, if $\boldsymbol{i} \neq \boldsymbol{j}$, then $\gamma_{k}(i) \cap \gamma_{k}(\boldsymbol{j})=\varnothing$, for $k<2$.

Using this notation we define an equivalence relation between models with added unary relations which we then show to be sufficient to guarantee the success of player II in the first-order Ehrenfeucht game.

Definition 6. $\left\langle\mathfrak{M}_{0}, \eta_{j}\right\rangle_{j<m} \mathscr{E}_{n}^{\prime}\left\langle\mathfrak{M}_{1}, \theta_{j}\right\rangle_{j<m}$ if and only if for each $\boldsymbol{i} \in \operatorname{Seq}\left(2^{m}, n\right)$, $\# \gamma_{0}(i) E_{n} \# \gamma_{1}(i)$.

THEOREM 3. I $f\left\langle\mathfrak{M}_{0}, \eta_{j}\right\rangle_{j<m} \mathscr{E}_{n}^{\prime}\left\langle\mathfrak{M}_{1}, \theta_{j}\right\rangle_{j<m}$, then $\left\langle\mathfrak{M}_{0}, \eta_{j}\right\rangle_{j<m} \equiv .\left\langle\mathfrak{M}_{1}, \theta_{j}\right\rangle_{j<m}$.

ProOF. The proof consists of providing a strategy by which player II can always win the game $\mathfrak{G}_{n}\left(\mathfrak{M}_{0}, \mathfrak{M}_{1}\right)$. Essentially, at each move player II matches (modulo $E_{n}$ ) the cardinality of the intersection of player I's choice with each of 
the $2^{m}$ Boolean combinations of the specified unary relations. That this is possible results from the hypothesis of the lemma.

Assume $k<n$ and $x_{0} \leftrightarrow y_{0}, \cdots, x_{k-1} \leftrightarrow y_{k-1}$ have been chosen at the previous moves to satisfy the following inductive hypothesis: for each $j<k$,

(a) for each $i<k, x_{i}=x_{j}$ if and only if $y_{i}=y_{j}$;

(b) for each $i<k, x_{i} \sim x_{j}$ if and only if $y_{i} \sim y_{j}$;

(c) there is a sequence $i_{j} \in \operatorname{Seq}\left(2^{m}, n\right)$ such that

$$
\left[x_{j}\right] \in \gamma_{0}\left(\boldsymbol{i}_{j}\right) \text { and }\left[y_{j}\right] \in \gamma_{1}\left(\boldsymbol{i}_{j}\right) ; \text { and }
$$

(d) for $l<2^{m}, x_{j} \in \Pi_{0}(l)$ if and only if $y_{j} \in \Pi_{1}(l)$.

Of course, for the basis step $(k=0)$ this assumption is vacuous.

Assume player I selects $x_{k} \in \mathfrak{M}_{0}$ (the case when player I selects $y_{k} \in \mathfrak{M}_{1}$ is similar). We show how player II selects $y_{k} \in M_{1}$ in such a way that the induction hypothesis again holds. Let $l<2^{m}$ be the unique integer such that $x_{k} \in \Pi_{0}(l)$.

CASE 1. $x_{k}=x_{j}$ for some $j<k$. Set $y_{k}=y_{j}$.

CASE 2. $x_{k} \in\left[x_{j}\right]$ for some $j<k$, but $x_{k} \notin\left\{x_{i} \mid i<k\right\}$.

By part (c) of the inductive hypothesis, $\#\left(\left[x_{j}\right] \cap \Pi_{0}(l)\right) E_{n} \#\left(\left[y_{j}\right] \cap \Pi_{1}(l)\right)$. Thus there must be a $y \in\left(\left[y_{j}\right] \cap \Pi_{1}(l)\right)-\left\{y_{i} \mid i<k\right\}$. Let $y_{k}$ be that $y$, and set $i_{k}=i_{j}$.

CASE 3. $x_{k} \notin\left[x_{j}\right]$ for each $j<k$; hence $\left[x_{k}\right] \cap \bigcup_{j<k}\left[x_{j}\right]=\varnothing$. Let $\boldsymbol{i}_{k}$ be such that $\left[x_{k}\right] \in \gamma_{0}\left(i_{k}\right)$. By assumption, $\# \gamma_{0}\left(i_{k}\right) E_{n} \# \gamma_{1}\left(i_{k}\right)$, so there must be a $[y]$ such that $[y] \in \gamma_{1}\left(i_{k}\right)$ and $[y] \cap \bigcup_{j<k}\left[y_{j}\right]=\varnothing$. Let $y_{k} \in[y] \cap \Pi_{1}(l)$. There must be such a $y_{k}$, since $x_{k} \in \Pi_{0}(l)$ and $\#\left(\left[x_{k}\right] \cap \Pi_{0}(l)\right) E_{n} \#\left([y] \cap \Pi_{1}(l)\right)$.

Clearly, after all $n$ moves, the map determined by $x_{k} \mapsto y_{k}$ is an isomorphism between $\left\{x_{k} \mid k<n\right\}$ and $\left\{y_{k} \mid k<n\right\}$ with respect to the equivalence relation and the specified unary relations, all restricted to these sets. Thus player II can always win $\mathfrak{G}_{n}\left(\mathfrak{M}_{0}, \mathfrak{M}_{1}\right)$; i.e., $\mathfrak{M}_{0} \equiv_{n} \mathfrak{M}_{1}$.

CoROllaRY 1. If $\mathfrak{M}_{0} \mathscr{E}_{n} \mathfrak{M}_{1}$ then $\mathfrak{M}_{0} \equiv_{n} \mathfrak{M}_{1}$.

PROOF. This follows simply by noting that $\mathscr{E}_{n}^{\prime}$ extends $\mathscr{E}_{n}$; i.e., $\mathfrak{M}_{0} \mathscr{E}_{n} \mathfrak{M}_{1}$ if and only if $\left\langle\mathfrak{M}_{0}, \eta_{j}\right\rangle_{j<0} \mathscr{E}_{n}^{\prime}\left\langle\mathfrak{M}_{1}, \theta_{j}\right\rangle_{j<0}$.

As we shall point out later, it is possible to use this corollary to prove directly that the first-order theory of an equivalence relation is decidable. Next, however, we prove a lemma that allows us to provide a strategy for player II for the secondorder Ehrenfeucht game.

LEMMA 1. For the primitive recursive function $g(m, n)=n(n+1)^{2^{m+1}}$, if $\left\langle\mathfrak{M}_{0}, \eta_{j}\right\rangle_{j<m} \mathscr{E}_{g(m, n)}^{\prime}\left\langle\mathfrak{M}_{1}, \theta_{j}\right\rangle_{j<m}$, then for each $\eta_{m} \subseteq \mathfrak{M}_{0}$ there is a $\theta_{m} \subseteq \mathfrak{M}_{1}$ such that $\left\langle\mathfrak{M}_{0}, \eta_{j}\right\rangle_{j<m+1} \mathscr{E}_{n}^{\prime}\left\langle\mathfrak{M}_{1}, \theta_{j}\right\rangle_{j<m+1}$.

Proof. Assuming that $\eta_{m} \subseteq \mathfrak{M}_{0}$ has been selected, we show how to construct 
a set $\theta_{m} \subseteq \mathfrak{M}_{1}$ that satisfies the requirements of the lemma. The construction consists of two phases. The first phase is to ensure that $\theta_{m}$ and $\bar{\theta}_{m}$ are big enough; the second, to ensure that $\bar{\theta}_{m}$ is small enough.

In phase $\mathrm{I}$, we construct $\delta_{\boldsymbol{j}}$ for each $\boldsymbol{j} \in \operatorname{Seq}\left(2^{m+1}, n\right)$. At the end of phase I, we consider what would be incorrect if $\theta_{m}$ were to equal $\bigcup\left\{\delta_{j} \mid j \in \operatorname{Seq}\left(2^{m+1}, n\right)\right\}$. In phase II we construct $\zeta_{j}$ to adjust $\delta_{j}$ to correct this error.

PHASE I. At the beginning, we may describe each equivalence class in $\mathfrak{M}_{0}$ and $\mathfrak{M}_{1}$ as unused. During the course of the construction, various equivalence classes will have their status changed to used. Consider in turn each $j \in \operatorname{Seq}\left(2^{m+1}, n\right)$. Let $N=N(j)=\min \left(n, \# \gamma_{0}(j)\right)$. Select $N$ distinct unused equivalence classes from $\gamma_{0}(j)$ and call them $\left[x_{0}\right], \cdots,\left[x_{N-1}\right]$. These unused equivalence classes must exist, since $N \leqq \# \gamma_{0}(\boldsymbol{j})$; and as remarked above, for $\boldsymbol{i}, \boldsymbol{j} \in \operatorname{Seq}\left(2^{m+1}, n\right), \boldsymbol{i} \neq \boldsymbol{j}$, $\gamma_{0}(\boldsymbol{i}) \cap \gamma_{0}(\boldsymbol{j})=\varnothing$; thus no other part of phase I can use any equivalence class in $\gamma_{0}(i)$. These $\left[x_{k}\right]_{k<N}$ are now used. For each $k<N$, let $\boldsymbol{i}_{k}$ be that element of Seq $\left(2^{m}, g(m, n)\right)$ satisfying $\left[x_{k}\right] \in \gamma_{0}\left[i_{k}\right]$. Pick distinct unused $\left[y_{k}\right] \in \gamma_{1}\left(i_{k}\right)$, for each $k<N$, and let $Y=\left\{\left[y_{k}\right] \mid k<N\right\}$. This can be done, since $\# \gamma_{0}\left(i_{k}\right) E_{g(m, n)} \# \gamma_{1}\left(i_{k}\right)$ by assumption, and there are at most $(n+1)^{2^{m+1}}$ elements of Seq $\left(2^{m+1}, n\right)$, and each element can cause at most $n$ equivalence classes to be used. The equivalence classes in $Y$ are now used.

For each $k<N$, consider the $2^{m}$ partition sets $\left\{\Pi_{0}(l) \mid l<2^{m}\right\}$. Modulo the equivalence relation $E_{g(m, n)}$, the cardinality of $\left[x_{k}\right] \cap \Pi_{0}(l)$ is the information of $i_{k}(l)$. Now consider the result of selecting $\eta_{m}$. This splits entry $l$ of $i_{k}$ into entries $j(2 l)$ and $j(2 l+1)$, where because of the enumerations assigned to $f_{l}^{(m)}, f_{2 l}^{(m+1)}$, and $f_{2 l+1}^{(m+1)}$, these are the cardinalities (modulo $E_{n}$ ) of $\left[x_{k}\right] \cap \Pi_{0}(l)$ $\cap \eta_{m}$ and $\left[x_{k}\right] \cap \Pi_{0}(l) \cap \bar{\eta}_{m}$ respectively. The idea is now to "carve up" the $\left[y_{k}\right]$ 's in $Y$, putting some pieces into $\theta_{m}$, in the same way the $\left[x_{k}\right]$ 's were carved up by $\eta_{m}$. For each $[y] \in Y$ consider the $2^{m}$ partition sets $\left\{\Pi_{1}(l) \mid l<2^{m}\right\}$. Within each of these, consider $[y] \cap \Pi_{1}(l)$. We carve this into two pieces $\alpha([y], l)$ and $\beta([y], l)$ with cardinalities $a$ and $b$ respectively. I.e., we will have

$$
\begin{gathered}
{[y] \cap \Pi_{1}(l)=\alpha([y], l) \cup \beta([y], l), \alpha([y], l) \cap \beta([y], l)=\varnothing,} \\
\# \alpha([y], l)=a
\end{gathered}
$$

and

$$
\# \beta([y], l)=b .
$$

This will be done in such a way that $a E_{n} j(2 l)$ and $b E_{n} j(2 l+1)$, for $l<2^{m}$. There are two cases:

(i) Unless $j(2 l)=n$ and $j(2 l+1)<n$, put $j(2 l)$ elements into $\alpha([y], l)$, and leave the remainder of $[y] \cap \Pi_{1}(l)$ in $\beta([y], l)$,

(ii) If $\boldsymbol{j}(2 l)=n$ and $\boldsymbol{j}(2 l+1)<n$, then put $\boldsymbol{j}(2 l+1)$ elements into $\beta([y], l)$ and leave the remainder of $[y] \cap \Pi_{1}(l)$ in $\alpha([y], l)$. 
After doing this for each $[y] \in Y$ and each $l<2^{m}$, set $\delta_{j}=\bigcup_{[y] \in Y} \bigcup_{l<2^{m}}$ $\alpha([y], l)$. This ends phase I.

If, in the course of phase $I$, all the equivalence classes of $\mathfrak{M}_{1}$ had been used, it would be sufficient to let $\theta_{m}=\bigcup\left\{\delta_{j} \mid \boldsymbol{j} \in \operatorname{Seq}\left(2^{m+1}, n\right)\right\}$. However, in general there remain unused equivalence classes; and because of these, if we were to use the above $\theta_{m}$, it might happen that $\# \gamma_{0}(j)<\# \gamma_{1}(j)$ modulo $E_{n}$, for some $\boldsymbol{j} \in \operatorname{Seq}\left(2^{m+1}, n\right)$. Such a $\boldsymbol{j}$ would have to satisfy $\forall l<2^{m}(\boldsymbol{j}(2 l)=0)$ since the problem concerns equivalence classes which were unused and hence would be subsets of $\bar{\theta}_{m}$. The solution is to consider those $i \in \operatorname{Seq}\left(2^{m}, g(m, n)\right)$ corresponding to the partition derived from the Boolean combinations of $\eta_{0}, \cdots, \eta_{m-1}$ which could give rise to this $\boldsymbol{j}$ when $\eta_{\boldsymbol{m}}$ was added. These $\boldsymbol{i}$ will be called the ancestors of $\boldsymbol{j}$. We then consider the set of sequences in $\operatorname{Seq}\left(2^{m+1}, n\right)$ which could have resulted from $i$ by the addition of some $\eta_{m}^{\prime}$ to $\eta_{k}$, for $k<m$. These are called the progeny of $\boldsymbol{i}$. We then show that any ancestor $\boldsymbol{i}$ with unused equivalence classes must have a $j_{0}$ among its progeny for which there are least $n$ equivalence classes in $\gamma_{0}\left(\boldsymbol{j}_{0}\right)$; and thus, using the "carving up" process described above, we may put all the unused equivalence classes of $i$ into $\gamma_{1}\left(j_{0}\right)$ and still have $\# \gamma_{0}\left(j_{0}\right) E_{n} \# \gamma_{1}\left(j_{0}\right)$. With this as an intuitive guide we describe the details below.

Phase II. Let $\theta^{\prime}=\bigcup\left\{\delta_{\boldsymbol{j}} \mid \boldsymbol{j} \in \operatorname{Seq}\left(2^{m+1}, n\right)\right\}$. For $\boldsymbol{j} \in \operatorname{Seq}\left(2^{m+1}, n\right)$, let $A(\boldsymbol{j})=$ ancestors of $\boldsymbol{j}=$

$$
\begin{gathered}
\left\{i \in \operatorname{Seq}\left(2^{m}, g(m, n)\right) \mid \forall l<2^{m}[((j(2 l) \neq n \& j(2 l+1) \neq n) \rightarrow j(2 l)+j(2 l+1)=\right. \\
i(l) \&((j(2 l)=n \vee j(2 l+1)=n) \rightarrow j(2 l)+j(2 l+1) \leqq i(l))]\} .
\end{gathered}
$$

Let $P(\boldsymbol{i})=$ progeny of $\left.\boldsymbol{i}=\left\{\boldsymbol{j} \in \operatorname{Seq}\left(2^{m+1}, n\right) \mid \boldsymbol{i} \in A(\boldsymbol{j})\right)\right\}$, and let $\gamma^{\prime}(\boldsymbol{j})$, for $\boldsymbol{j} \in \operatorname{Seq}\left(2^{m+1}, n\right)$, characterize the cardinalities of equivalence classes intersected with the Boolean combinations of $\theta_{0}, \cdots, \theta_{m-1}, \theta^{\prime}$. Set $S=\left\{j \in \operatorname{Seq}\left(2^{m+1}, n\right)\right\}$ $\left.\neg\left[\# \gamma_{0}(j) E_{n} \# \gamma^{\prime}(j)\right]\right\}$. For each $\boldsymbol{j} \in \operatorname{Seq}\left(2^{m+1}, n\right)$ set $\zeta_{j}=\varnothing$ initially. Some $\zeta_{j}$ will be modified (perhaps more than once) in carrying out the following construction. For each $\boldsymbol{j} \in S$ do the following. Examine each $\boldsymbol{i} \in A(\boldsymbol{j})$. If there are no unused $[y] \in \gamma_{1}[i]$, then there is nothing to do. However, for each $i \in A(j)$ which contains equivalence classes which have not yet been used, find a $j_{0} \in P(i)$ such that $\# \gamma_{0}\left(j_{0}\right) \geqq n$. Such a sequence must exist by the following cardinality argument. Assume no such $\boldsymbol{j}_{0}$ exists. Note that $\bigcup\left\{\gamma_{0}\left(\boldsymbol{j}_{1}\right) \mid \boldsymbol{j}_{1} \in P(\boldsymbol{i})\right\} \supseteq \gamma_{0}(\boldsymbol{i})$, and that the union is a disjoint union. Thus, $\Sigma_{\boldsymbol{j}, \in P(i)} \# \gamma_{0}\left(\boldsymbol{j}_{1}\right) \geqq \# \gamma_{0}(\boldsymbol{i})$. But $P(i) \subseteq \operatorname{Seq}\left(2^{m+1}, n\right)$, so $\# P(i) \leqq(n+1)^{2^{m+1}}$. Thus we have

$$
g(m, n)=n(n+1)^{2^{m+1}}>\sum_{j_{1} \in P(i)} \# \gamma_{0}\left(j_{1}\right) \geqq \# \gamma_{0}(i) .
$$

Since $\left\langle\mathfrak{M}_{0}, \eta_{j}\right\rangle_{j<m} \mathscr{E}_{g(m n)}^{\prime}\left\langle\mathfrak{M}_{1}, \theta_{j}\right\rangle_{j<m}$, this implies that $\# \gamma_{0}(i)=\# \gamma_{1}(i)$. By the construction in phase I, since $\# \gamma_{0}\left(j_{1}\right)<n$ for each $j_{1} \in P(i)$, all the $[x] \in \gamma_{0}(i)$ must have been used. Furthermore, just as many elements of $\gamma_{1}(i)$ must have 
been used as were used from $\gamma_{0}(i)$. But this implies that there were no unused $[y] \in \gamma_{1}(i)$ which contradicts the selection of $i$. Let $Y=\left\{[y] \mid[y] \in \gamma_{1}(i)\right.$ and $[y]$ is unused $\}$. The equivalence classes in $Y$ are now used. Modify $\zeta_{j_{0}}$ by carving up the $[y] \in Y$ as in phase I. I.e., $\zeta_{j_{0}}:=\zeta_{j_{0}} \cup \bigcup_{[y] \in Y} \bigcup_{l<2^{m}} \alpha([y], l)$, where $\alpha([y], l)$ is formed as in phase $\mathrm{I}$, and the symbol $:=$ represents replacement.

It should be clear that after this has been done for each $j \in S$, then setting $\theta_{m}=\theta^{\prime} \cup \cup\left\{\zeta_{j} \in \operatorname{Seq}\left(2^{m+1}, n\right)\right\}$ gives the required $\theta_{m}$.

THEOREM 4. Let $g(k, n)=n(n+1)^{2^{k+1}}$, and let $h(k, n)$ be the primitive recursive function defined by $h(0, n)=n$ and $h(k+1, n)=g(k, h(k, n))$. Then $\left\langle\mathfrak{M}_{0}, \eta_{j}\right\rangle_{j<m} \mathscr{E}_{h(k, n)}^{\prime}\left\langle\mathfrak{M}_{1}, \theta_{j}\right\rangle_{j<m}$ implies that $\left\langle\mathfrak{M}_{0}, \eta_{j}\right\rangle_{j<m} \equiv_{k, n}\left\langle\mathfrak{M}_{1}, \theta_{j}\right\rangle_{j<m}$.

Proof. By induction on $k$.

For $k=0$, the result is merely a restatement of Theorem 3 .

Assume the result holds for some $k$, and assume that $\left\langle\mathfrak{M}_{0}, \eta_{j}\right\rangle_{j<m} \mathscr{E}_{h(k+1 . n)}^{\prime}$ $\left\langle\mathfrak{M}_{1}, \theta_{j}\right\rangle_{j<m}$. Thus $\left\langle\mathfrak{M}_{0}, \eta_{j}\right\rangle_{j<m} \mathscr{E}_{g(m, h(k, n))}^{\prime}\left\langle\mathfrak{M}_{1}, \theta_{j}\right\rangle_{j<m}$. Lemma 1, the inductive hypothesis, and the definition of $k, n$-equivalence suffice to show that

$$
\left\langle\mathfrak{M}_{0}, \eta_{j}\right\rangle_{j<m} \equiv_{k+1, n}\left\langle\mathfrak{M}_{1}, \theta_{j}\right\rangle_{j<m}
$$

THEOREM 5. The monadic second-order theory of an equivalence relation is decidable by a primitive recursive procedure.

PROOF. It follows fromstandard techniques of second-order logic that any sentence is logically equivalent to one in prenex form where all the second-order quantifiers precede all the first-order quantifiers. By Theorems 1 and 4, to check the validity of a sentence

$$
\psi=Q_{0} \xi_{0} \cdots Q_{m-1} \xi_{m-1} Q_{0} v_{0} \cdots Q_{n-1} v_{n-1} \phi\left(\xi_{0}, \cdots, \xi_{m-1}, v_{0}, \cdots, v_{n-1}\right)
$$

in which $\varphi$ is quantifier free, it is sufficient to verify that $\mathfrak{M} \vDash \psi$ for those $\mathfrak{M}$ having at most $h(m, n)$ equivalence classes of each cardinality less than or equal to $h(m, n)$ and no equivalence classes of larger cardinalities. There are at most $(h(m, n)+1)^{h(m, n)}-1$ of these models, each with at most $\frac{1}{2} h(m, n)^{2}(h(m, n)+1)$ elements. This simple veritication can be done by a primitive recursive procedure (cf. Nerode).

It should be noted that the decision procedure itself does not require that the sentence being decided be converted to an equivalent normal form, only that a bound be calculated based on the number of quantifiers the equivalent form would contain.

COROLLARY 2. The first-order theory of a equivalence relation is decidable.

PROOF. This follows immediately from Theorem 5. However, a more direct proof is obtained by applying the method of the proof of Theorem 5 to the bound given in Theorem 3 . 


\section{References}

A. Ehrenfeucht (1961), 'An application of games to the completeness problem for formalized theories', Fundamenta Mathematicae 49, 129-141.

A. Janiczak (1953), 'Undecidability of some simple formalized theories', Fundamenta Mathematicae, 40, 131-139.

John J. Le Torneau (1968), Decision Problems Related to the Concept of Operation (Ph.D. Thesis, Berkeley, 1968).

Anil Nerode, Axiomatic Theories and Recursion Theory (unpublished).

Michael O. Rabin (1969), 'Decidability of second-order theories and automata on infinite trees' Transactions of the American Mathematical Society 141, 1-35.

University of Massachusetts

Computer and Information Science

Amherst 01002

U.S.A. 